\title{
Formalismo de Hamilton-Jacobi à la Carathéodory
}

(The Hamilton-Jacobi formalism a la Carathéodory)

\author{
M.C. Bertin ${ }^{1}$, B.M. Pimentel ${ }^{1}$ e P.J. Pompeia ${ }^{2}$ \\ ${ }^{1}$ Instituto de Física Teórica, Universidade Estadual Paulista, São Paulo, SP, Brasil \\ ${ }^{2}$ Comando-Geral de Tecnologia Aeroespacial, Instituto de Fomento e Coordenação Industrial, S. J. dos Campos, SP, Brasil \\ Recebido em 13/3/2007; Aceito em 21/6/2007
}

\begin{abstract}
Aqui traremos a descrição do formalismo de Hamilton-Jacobi para sistemas regulares como desenvolvido no livro de Carathéodory, seguida por dois exemplos que mostram sistematicamente sua aplicabilidade. Palavras-chave: equação de Hamilton-Jacobi, sistemas regulares.
\end{abstract}

We will bring the description of the Hamilton-Jacobi formalism for regular systems as developed in Carathéodory's book, followed by two examples that show its applicability.

Keywords: Hamilton-Jacobi equation, regular systems.

\section{Introdução}

Em quatro artigos monumentais publicados em 1926 [1], Schrödinger inaugura a mecânica ondulatória, na qual a quantização é abordada como um problema de resolução de equações de autovalores. Em conjunto com a mecânica de Heisenberg e os trabalhos de Dirac, esses artigos edificam as bases teóricas da mecânica quântica. Quase um século antes, Hamilton mostra seu formalismo para a mecânica clássica baseado na analogia óptico-mecânica, que relaciona a óptica geométrica à mecânica analítica. Como legítimo sucessor de Hamilton, Schrödinger utiliza-se da mesma construção analógica, que permitiu a abertura de novas fronteiras da física.

$\mathrm{Na}$ introdução do segundo de sua série de artigos, Schrödinger escreve sobre Hamilton:

Infelizmente esta concepção significativa e poderosa de Hamilton é privada, nas reproduções mais modernas, de sua bela vestimenta, como um acessório supérfluo, em favor de uma representação mais colorida da correspondência analítica.

Sobre os trabalhos de Schrödinger, o mesmo poderia ser dito nos dias de hoje sem qualquer alteração.

A analogia óptico-mecânica está ligada ao fato de ambas as disciplinas, a óptica e a mecânica, serem deduzidas por princípios variacionais (para uma literatura histórica sobre o cálculo variacional, veja a Ref. [2]). E a primeira ligação foi estabelecida quando Hamilton

\footnotetext{
${ }^{1}$ E-mail: pimentel@ift.unesp.br.

percebeu que seu princípio é equivalente ao de Fermat, tendo como resultado que trajetórias dos raios de luz no espaço euclidiano são ligadas às trajetórias dinâmicas no espaço de configuração por um formalismo matemático comum.

Enquanto a mecânica clássica estaria ligada à óptica geométrica, a mecânica quântica estaria conectada à óptica ondulatória. O limite estaria na capacidade da definição de trajetórias, que só é possível na óptica geométrica, onde consideramos que a trajetória tem dimensões muito maiores que o comprimento de onda. $\mathrm{O}$ conceito de trajetória não faz sentido na teoria ondulatória, na qual o comprimento de onda é grande em relação ao "caminho da luz". Considerou-se, então, natural que nos sistemas quânticos a mecânica clássica não funcionasse, tanto quanto a óptica geométrica não poderia ser usada para problemas que envolvessem, por exemplo, a difração.

Existe um formalismo, nascido da mecânica de Hamilton e da teoria das transformações canônicas de Jacobi, que é o formalismo comum entre ambas as mecânicas e a óptica. É o formalismo de HamiltonJacobi, (HJ). Este formalismo tornou-se o ponto de partida de Schrödinger para a formulação da mecânica quântica. Neste artigo, nos dedicaremos a apresentar este formalismo sobre o geometricamente rico escopo de Carathéodory [3].

O formalismo de HJ é baseado na analogia entre três disciplinas matemáticas que por algum tempo foram tidas como independentes: a teoria das equações diferenciais ordinárias (EDO), a teoria das equações di- 
ferenciais parciais (EDP) e o cálculo variacional. Esta analogia tem uma forte interpretação geométrica, que Carathéodory chama pelo sugestivo nome de quadro completo (complete figure). Nos textos usuais sobre mecânica clássica [4-9] a equação de HJ é vinculada ao formalismo Hamiltoniano através das transformações canônicas. Neste ponto de vista, a equação de HJ surge ao considerarmos o problema de se encontrar uma transformação no espaço de fase capaz de levar a resolução das equações dinâmicas de Hamilton à forma mais elementar possível. O formalismo é tratado, então, como um mero meio para se encontrar soluções para as equações de Hamilton. No quadro completo, assim mostraremos, a análise de HJ é independente da abordagem Hamiltoniana da mecânica, consistindo em uma teoria completa, auto-suficiente e com resultados muito gerais.

Neste trabalho estudaremos o formalismo de HJ para sistemas regulares, que obedecem à condição Hes$\operatorname{siana}^{2}$

$$
\operatorname{det} W \neq 0 \quad w_{i j} \equiv \frac{\partial^{2} L}{\partial \dot{q}^{i} \partial \dot{q}^{j}},
$$

em que $W$ é a matriz Hessiana com elementos $w_{i j}$. Mostraremos como a equação de HJ pode ser obtida sem a passagem ao formalismo Hamiltoniano, a partir do princípio de ação estacionária. Ilustraremos também o método de Cauchy, também chamado método das características, para resolver a equação de HJ. A partir dessa análise seremos capazes de definir um espaço de fase e, neste, encontrar os parênteses de Poisson que descrevem a evolução temporal de observáveis. Analisaremos as propriedades das soluções e resolveremos dois problemas simples a partir do método de separação de variáveis.

\section{Lagrangeanas equivalentes}

Vamos começar com o tradicional enunciado do princípio de ação estacionária de Hamilton. Dada uma curva $C$ no espaço de configuração caracterizada pelas equações paramétricas

$$
q^{i}=q^{i}(t)
$$

e uma função de Lagrange $L(t, q, \dot{q})$ referente a um sis- tema de $n$ graus de liberdade, definimos a integral

$$
A_{[C]}=\int_{t_{1}}^{t_{2}} L\left(t, q^{i}, \dot{q}^{i}\right) d t \quad i \in\{1, \ldots, n\},
$$

calculada sobre $C$ entre dois pontos fixos $t_{1}$ e $t_{2}$. Esta integral é chamada de ação e vem a ser um funcional: relaciona curvas do espaço de configuração a números reais.

Vamos agora considerar a existência de uma curva $\bar{C}$ em uma vizinhança fechada ${ }^{3}$ de $C$, que se interceptam nos pontos $t_{1}$ e $t_{2}$. Para $\bar{C}$ podemos calcular a integral

$$
\bar{A}_{[\bar{C}]}=\int_{t_{1}}^{t_{2}} L\left(t, \bar{q}^{i}, \frac{d \bar{q}^{i}}{d t}\right) d t .
$$

A curva $\bar{C}$ é chamada curva de comparação (Fig. 1). Vamos estabelecer que $C$ seja a trajetória natural do sistema e que a integral da ação calculada sobre esta curva, $A_{[C]}$, seja sempre um máximo ou um mínimo relativo a uma curva de comparação qualquer na vizinhança de $C$. Ou seja, para qualquer $\bar{C}$,

$$
A_{[C]}>\bar{A}_{[\bar{C}]} \quad \text { ou } \quad A_{[C]}<\bar{A}_{[\bar{C}]}
$$

Esta é a condição para que a curva $C$ seja um extremo de $A$. Com relação a outros princípios formulados anteriormente, o princípio de Hamilton tem uma vantagem importante. É estabelecido também para problemas não conservativos, para os quais a ação é dependente explicitamente do tempo. O princípio de ação estacionária não necessita de condições subsidiárias e exige apenas que a integral seja calculada entre dois pontos fixos da trajetória.

O problema básico do cálculo variacional é encontrar a forma paramétrica da trajetória do sistema no espaço de configuração, $q^{i}=q^{i}(t)$, de modo que a primeira variação da ação seja nula. A abordagem direta a esse problema leva às equações de Euler-Lagrange, mas desejamos seguir por outro caminho. Note em primeiro lugar que se construirmos uma segunda função Lagrangiana com a forma

$$
\bar{L}=L-\frac{d S}{d t}
$$

na qual $S$ é uma função apenas das coordenadas e do

\footnotetext{
${ }^{2}$ No formalismo hamiltoniano, a dedução das equações canônicas de Hamilton dependem desta condição.

${ }^{3}$ Dada a curva $C$ definida pela Eq. (1), uma segunda curva $\bar{C}$, cujas equações paramétricas são $q^{i}=\bar{q}^{i}(t)$, pertence a uma vizinhança fechada $(\varepsilon, \eta)$ de $C$ se, primeiro, para todos os valores de $t$ no intervalo $t_{1} \leq t \leq t_{2}$, forem satisfeitas as condições

$$
\left|\bar{q}^{i}(t)-q^{i}(t)\right| \leq \varepsilon
$$

e, para todo valor de $t$ no mesmo intervalo para os quais existam as derivadas $d \bar{q}^{i} / d t$, forem também satisfeitas

Ver [3].

$$
\left|\frac{d \bar{q}^{i}}{d t}-\dot{q}^{i}\right| \leq \eta .
$$
}


tempo, essa segunda Lagrangiana leva à ação

$$
\begin{gathered}
\bar{A}=\int_{\bar{t}_{1}}^{\bar{t}^{2}} \bar{L} d t=\int_{\bar{t}_{1}}^{\bar{t}^{2}} L d t-\int_{\bar{t}_{1}}^{\bar{t}^{2}} \frac{d S}{d t} d t= \\
\int_{\bar{t}_{1}}^{\bar{t}^{2}} L d t-S\left(\bar{t}_{2}, q_{\bar{t}_{2}}\right)+S\left(\bar{t}_{1}, q_{\bar{t}_{1}}\right) .
\end{gathered}
$$

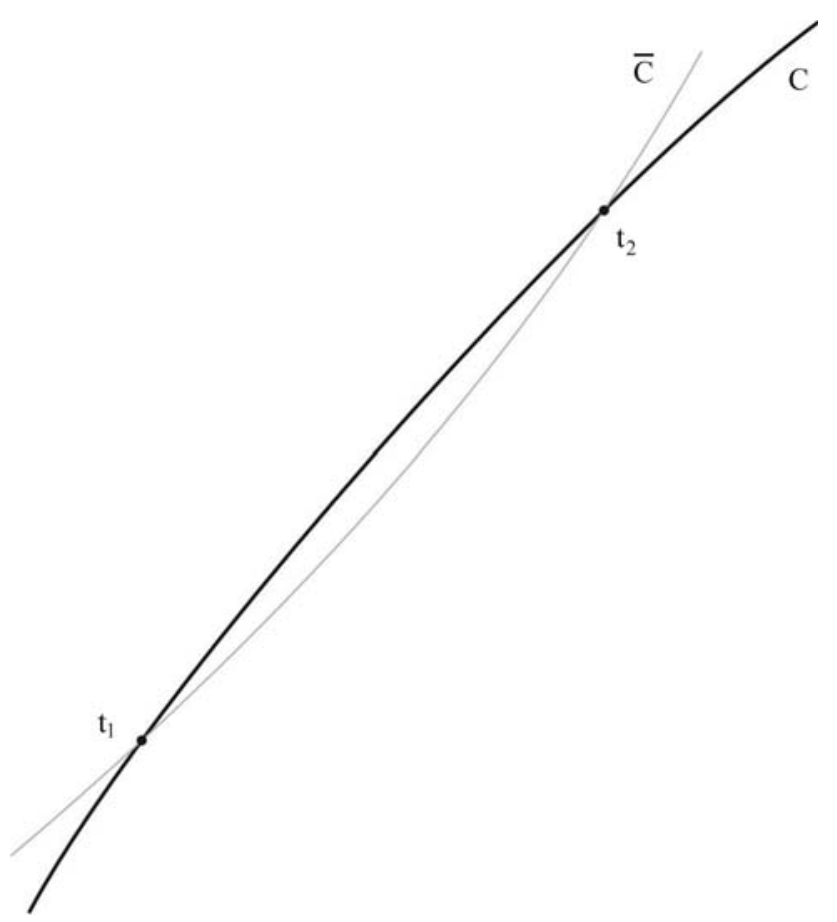

Figura 1 - A trajetória dinâmica e uma curva de comparação $\bar{C}$ em uma região do espaço de configuração.

Podemos fazer com que a ação $\bar{A}$ varie entre os mesmos valores de $A$, ou seja, vamos exigir que $\bar{t}_{1}=t_{1} \mathrm{e}$ $\bar{t}_{2}=t_{2}$. Se calcularmos a primeira variação ${ }^{4}$ na Eq. (6) entre os mesmos limites da Eq. (2), teremos como resultado

$$
\delta \bar{A}=\delta A-\delta S\left(t_{2}, q_{t_{2}}\right)+\delta S\left(t_{1}, q_{t_{1}}\right) .
$$

No entanto, $S$ é calculado nos pontos de fronteira, e sua variação é 5

$$
\delta S=\frac{\partial S}{\partial q^{i}} \delta q^{i} .
$$

Contudo, a ação é calculada entre pontos fixos, de modo que $\delta q$ é identicamente nulo. Assim, $\delta S=0$ e

$$
\delta \bar{A}=\delta A .
$$

Este resultado mostra que a trajetória que extremiza $\bar{A}$ é a mesma que extremiza $A$, de modo que dizemos que $\bar{L}$ e $L$ são Lagrangianas equivalentes. Levam ao mesmo problema variacional e dizem respeito à mesma dinâmica. $S$ vem a ser uma função geradora de

uma transformação de coordenadas no espaço de configuração, à qual a dinâmica é invariante.

A trajetória $q^{i}(t)$, solução do problema variacional, é também solução do sistema de EDO

$$
\dot{q}^{i}=\phi^{i}(t, q),
$$

se todos os $\phi^{i}$ existirem. Segundo Carathéodory, se pudermos encontrar as funções $\phi$, a condição suficiente para que $A$ seja estacionária está em encontrar uma função $S(t, q)$ para a qual

$$
\bar{L}(t, q, \phi)=0
$$

e, em qualquer curva na vizinhança de $C, \bar{L}>0$ ou $\bar{L}<0$.

\section{A equação de Hamilton-Jacobi}

Vamos agora proceder à procura da função $S$. Para que $C$ seja um extremo de $A$, devemos ter obedecida a condição

$$
\frac{\partial \bar{L}}{\partial \dot{q}^{i}}=0
$$

Esta condição nos levará a

$$
\begin{aligned}
& {\left[\frac{\partial L}{\partial \dot{q}^{i}}-\frac{\partial}{\partial \dot{q}^{i}} \frac{d S}{d t}\right]_{\dot{q}^{i}=\phi^{i}}=} \\
& {\left[\frac{\partial L}{\partial \dot{q}^{i}}-\frac{\partial}{\partial \dot{q}^{i}}\left(\frac{\partial S}{\partial q^{j}} \dot{q}^{j}\right)-\frac{\partial^{2} S}{\partial \dot{q}^{i} \partial t}\right]_{\dot{q}^{i}=\phi^{i}}=} \\
& {\left[\frac{\partial L}{\partial \dot{q}^{i}}-\frac{\partial^{2} S}{\partial \dot{q}^{i} \partial q^{j}} \dot{q}^{j}-\frac{\partial S}{\partial q^{j}} \frac{\partial \dot{q}^{j}}{\partial \dot{q}^{i}}-\frac{\partial^{2} S}{\partial \dot{q}^{i} \partial t}\right]_{\dot{q}^{i}=\phi^{i}}=0 .}
\end{aligned}
$$

Mas $S$ não depende de $\dot{q}$, de modo que os únicos elementos não nulos obedecem à relação

$$
\left[\frac{\partial L}{\partial \dot{q}^{i}}-\frac{\partial S}{\partial q^{j}} \frac{\partial \dot{q}^{j}}{\partial \dot{q}^{i}}\right]_{\dot{q}^{i}=\phi^{i}}=\left[\frac{\partial L}{\partial \dot{q}^{i}}-\frac{\partial S}{\partial q^{j}} \delta_{i}^{j}\right]_{\dot{q}^{i}=\phi^{i}}=0
$$

ou seja,

$$
p_{i} \equiv \frac{\partial L}{\partial \dot{q}^{i}}=\frac{\partial S}{\partial q^{i}} .
$$

Hora de uma pequena digressão para uma interpretação geométrica da função $S$. Para que a interpretação seja mais visível, vamos em princípio imaginar um sistema de partícula única em coordenadas cartesianas sem vínculos. As condições para este sistema são

$$
p_{x}=\frac{\partial S}{\partial x}, \quad p_{y}=\frac{\partial S}{\partial y}, \quad p_{z}=\frac{\partial S}{\partial z},
$$

$\mathrm{Ou}$

$$
\mathbf{p}=\nabla S
$$

\footnotetext{
${ }^{4} \mathrm{O}$ símbolo $\delta$ foi introduzido por Lagrange e representa a variação virtual de uma função.

${ }^{5}$ Neste trabalho usaremos a convenção de soma para os índices das coordenadas generalizadas. Quando aparecerem em duplas, somam-se os objetos em toda a extensão de valores do índice. Assim, $\frac{\partial S}{\partial q^{i}} \delta q^{i}$ significa na verdade $\sum_{i} \frac{\partial S}{\partial q^{i}} \delta q^{i}$.
} 
Este resultado nos faz pensar que $S(x, y, z)$ define uma família de superfícies $S=\sigma$, em que $\sigma$ é um parâmetro real. A condição (14) nos diz que o momento é transverso às superfícies definidas por $\sigma$ (Fig. 2). Se voltarmos para a situação de $n$ partículas, isto nos mostra que $S=\sigma$ vem a ser uma família de superfícies no espaço de configuração, e devem ser escolhidas de modo que a trajetória do sistema intercepte ortogonalmente os membros dessa família. ${ }^{6}$ Em cada ponto da trajetória passa uma única superfície, de modo que podemos usar o parâmetro da curva, neste caso o tempo, para diferenciar os valores do parâmetro $\sigma$.

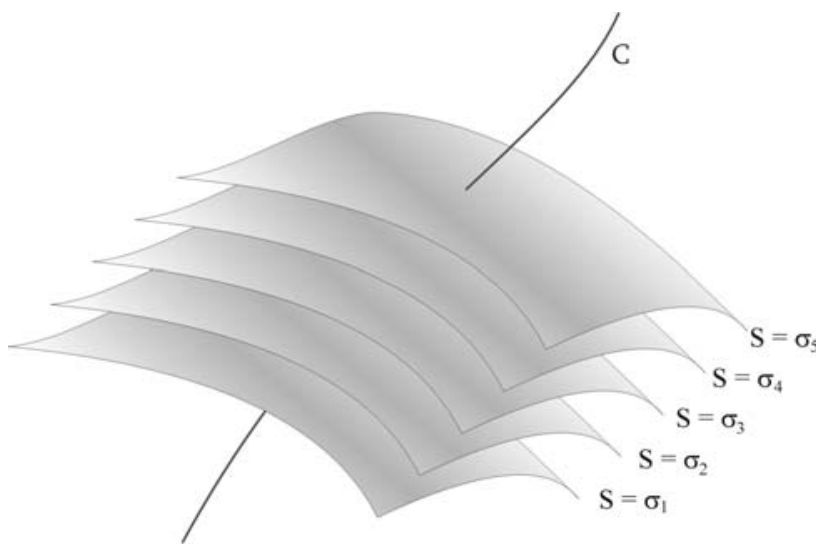

Figura 2 - Representação da trajetória e da família de superfícies.

Se a condição (10) é válida, então temos

$$
L=\frac{\partial S}{\partial t}+\dot{q}^{i} \frac{\partial S}{\partial q^{i}},
$$

ou seja,

$$
A=\int_{t_{1}}^{t_{2}} L d t=\int_{t_{1}}^{t_{2}}\left(\frac{\partial S}{\partial t}+\dot{q}^{i} \frac{\partial S}{\partial q^{i}}\right) d t=\sigma_{2}-\sigma_{1} .
$$

Então, a ação entre dois pontos de $C$ caracterizados por $t_{1}$ e $t_{2}$ é calculada pela diferença entre duas superfícies da família.

Mas a Eq. (12) não é a única condição que repousa sobre $S$. A partir da Eq. (10) vamos escrever

$$
L(t, q, \phi)-\frac{\partial}{\partial t} S(t, q)-\dot{q}^{i} \frac{\partial}{\partial q^{i}} S(t, q)=0 .
$$

Usando a Eq.(12), obtemos que

$$
\frac{\partial}{\partial t} S(t, q)+p_{i} \dot{q}^{i}-L(t, q, \dot{q})=0 .
$$

Essa equação pode nos fornecer uma equação diferencial para $S$, se pudermos eliminar as velocidades e escrevê-las em função das posições. Para tanto, temos a definição

$$
p_{i} \equiv \frac{\partial L}{\partial \dot{q}^{i}}
$$

\footnotetext{
${ }^{6}$ Esta interpretação tem um óbvio paralelo na teoria ondulatória da luz, onde o vetor de Poynting $\mathbf{S}$ deve ser sempre ortogonal à frente de onda. Essa analogia não é absolutamente casual, mas vem do fato de a teoria ondulatória também poder ser deduzida por princípios variacionais.
}

Se pudermos inverter essas equações e escrever as velocidades em função das posições e dos momentos, nosso objetivo será alcançado. Precisamos que seja satisfeita a condição

$$
\operatorname{det} W \neq 0 \quad w_{i j} \equiv \frac{\partial p_{i}}{\partial \dot{q}^{j}}=\frac{\partial^{2} L}{\partial \dot{q}^{i} \partial \dot{q}^{j}},
$$

que é a condição Hessiana. Sistemas que obedecem à condição Hessiana são chamados sistemas regulares. Obedecida essa condição, as velocidades podem ser escritas como funções dos momentos e das posições $\dot{q}^{i}=\eta^{i}(t, q, p)$ e, assim, podemos reescrever a Eq. (16) como

$$
\frac{\partial}{\partial t} S(t, q)+p_{i} \eta^{i}-L(t, q, \eta)=0 .
$$

Normalmente definimos nessa equação a função de Hamilton

$$
\begin{aligned}
& H(t, q, p) \equiv p_{i} \eta^{i}(t, q, p)-L(t, q, \eta)= \\
& H\left(t, q, \frac{\partial S}{\partial q}\right) .
\end{aligned}
$$

Temos a equação

$$
\frac{\partial}{\partial t} S(t, q)+H\left(t, q, \frac{\partial S}{\partial q}\right)=0
$$

Esta é uma equação diferencial parcial, que vem a ser a equação de Hamilton-Jacobi, (HJ). É a segunda condição sobre a família de superfícies para que a ação calculada sobre uma congruência de trajetórias $C$ transversal a essa família seja estacionária. Portanto, o problema de se encontrar equações de movimento através de extremos de um funcional nos leva a considerar essa família de superfícies, encontrada pela Eq. (19) e, através dela, encontrar a trajetória do sistema.

\section{Equações características}

Basta que, para um dado sistema cuja Lagrangiana é conhecida e cuja condição Hessiana seja satisfeita, resolvamos a equação de HJ para encontrar a família de superfícies $S$. No entanto, ainda não sabemos como encontrar a trajetória dinâmica a partir dessa informação. Existem duas formas de fazê-lo. A primeira não exige que se resolva a equação de HJ, o que pode ser uma grande vantagem pois, na maioria dos casos, a equação não é linear e encontrar uma solução, mesmo que seja parcial, pode não ser possível. Mas a equação depende apenas de primeiras derivadas de $S$, ou seja, é de primeira ordem. Equações diferenciais parciais de primeira ordem têm sempre um conjunto de equações diferenciais ordinárias relacionado. Podemos encontrar esse sistema de EDO pelo método das características, primeiro desenvolvido por Cauchy. 
Em cada ponto de $C$ podemos associar um campo $p_{i}$ que, já sabemos, satisfaz a condição de ser transversal à família de superfícies $S=\sigma$ :

$$
p_{i}=\frac{\partial S}{\partial q^{i}} .
$$

Esta condição nada nos diz sobre a dinâmica do sistema, ou seja, não é uma condição sobre sua evolução temporal. Contudo, traz informações sobre a direção da trajetória. A partir dela podemos construir

$$
\frac{d p_{i}}{d t}=\frac{\partial^{2} S}{\partial q^{i} \partial t}+\dot{q}^{j} \frac{\partial^{2} S}{\partial q^{i} \partial q^{j}} .
$$

Vamos também diferenciar a equação de HJ com relação a $q$,

$$
\frac{\partial H}{\partial q^{i}}=-\frac{\partial^{2} S}{\partial q^{i} \partial t}-\frac{\partial H}{\partial p_{j}} \frac{\partial^{2} S}{\partial q^{i} \partial q^{j}} .
$$

Note que, em função da definição da função Hamiltoniana (18), surge a identidade

$$
\frac{\partial H}{\partial p_{i}}=\dot{q}^{i}
$$

que inserida na Eq. (21) resulta em

$$
\frac{\partial H}{\partial q^{i}}=-\frac{\partial^{2} S}{\partial q^{i} \partial t}-\dot{q}^{j} \frac{\partial^{2} S}{\partial q^{i} \partial q^{j}} .
$$

Assim, comparando as Eqs. (20) e (23), em conjunto com a identidade (Eq. (22)), surgem as equações ordinárias de primeira ordem

$$
\dot{p}_{i}=-\frac{\partial H}{\partial q^{i}} \quad \text { e } \quad \dot{q}^{i}=\frac{\partial H}{\partial p_{i}} .
$$

Estas são $2 n$ das equações características da equação de HJ e podemos ver que coincidem com as equações canônicas do formalismo Hamiltoniano. Para encontrar a forma da Eq. (9), basta notarmos que a segunda equação canônica define exatamente este campo de velocidades, mas em função de $t, q$ e também de $p$. Ou seja,

$$
\dot{q}^{i}=f^{i}(t, q, p) .
$$

Podemos, contudo, utilizar a primeira equação para encontrar os momentos em função das posições e do tempo. Esta equação tem a forma $p_{i}=g^{i}(t, q)$ e, inserida na Eq. (25), resulta na forma desejada

$$
\dot{q}^{i}=\phi^{i}(t, q),
$$

cuja solução vem a ser a trajetória $C$ do sistema no espaço de configuração.

Outra equação característica surge com a diferenciação de $S$ :

$$
d S=\frac{\partial S}{\partial t} d t+\frac{\partial S}{\partial q^{i}} d q^{i}=-H d t+p_{i} d q^{i}
$$

ou, usando a segunda Eq. (24),

$$
\frac{d S}{d t}=p_{i} \frac{\partial H}{\partial p_{i}}-H
$$

Com este procedimento encontramos, a partir de uma equação diferencial parcial de primeira ordem, um sistema de $2 n+1$ equações ordinárias de primeira ordem

$$
\begin{aligned}
& d p_{i}=-\frac{\partial H}{\partial q^{i}} d t, \quad d q^{i}=\frac{\partial H}{\partial p_{i}} d t \quad \mathrm{e} \\
& d S=\left(p_{i} \frac{\partial H}{\partial p_{i}}-H\right) d t .
\end{aligned}
$$

Essas equações nos permitem definir o espaço de fase das variáveis $q$ e $p$, de modo que as soluções das equações canônicas de Hamilton dão origem também a curvas neste espaço de fase. A evolução dinâmica de uma função do espaço de fase, que é, geralmente, um observável físico, é dada por

$$
\frac{d f}{d t}=\frac{\partial f}{\partial q^{i}} \dot{q}^{i}+\frac{\partial f}{\partial p_{i}} \dot{p}_{i}=\frac{\partial f}{\partial q^{i}} \frac{\partial H}{\partial p_{i}}-\frac{\partial f}{\partial p_{i}} \frac{\partial H}{\partial q^{i}} .
$$

Do lado direito da equação anterior está uma estrutura antissimétrica, os parênteses de Poisson (PP). Os PP de duas funções $f(q, p)$ e $g(q, p)$ no espaço de fase são definidos por

$$
\{f, g\}=\frac{\partial f}{\partial q^{i}} \frac{\partial g}{\partial p_{i}}-\frac{\partial f}{\partial p_{i}} \frac{\partial g}{\partial q^{i}}
$$

Assim, a evolução dinâmica de observáveis pode ser escrita por $^{7}$

$$
\frac{d f}{d t}=\{f, H\}
$$

Portanto, recuperamos o formalismo Hamiltoniano a partir da equação de HJ, passo que fecha a dedução do quadro completo de Carathéodory. O quadro completo é um conjunto de relações. Começamos com um problema variacional: quais são as condições para que uma determinada trajetória seja um extremo de um determinado funcional? Esse problema nos levou, no caso de sistemas regulares, a uma equação diferencial parcial de primeira ordem. Uma solução dessa equação representa uma família de superfícies no espaço de configuração que é ortogonal à trajetória.

Já a trajetória pode ser encontrada pela resolução das equações características com condições iniciais apropriadas. Essas equações dão origem não apenas a uma curva, mas a todo um conjunto de curvas que não se interceptam no espaço de fase. Esse conjunto é chamado de Congruência e todos os seus membros devem ser ortogonais a todos os membros da família de superfícies. Em razão desse sistema de relações, chamaremos a congruência de curvas características.

\footnotetext{
${ }^{7}$ Se $f$ depender explicitamente de $t$ devemos acrescentar a esta próxima equação o termo $\partial f / \partial t$.
} 


\section{Propriedades das soluções}

Existem dois métodos para se resolver um sistema e encontrar sua trajetória no espaço de configuração. O primeiro é resolver as duas primeiras equações do sistema (29), que são as equações canônicas de Hamilton. Se escolhermos este caminho, o problema de se resolver a equação de HJ está em empregar o formalismo Hamiltoniano. Outra forma é tentar encontrar uma solução da equação de HJ (19).

No geral, a equação de HJ é não linear. Não há um teorema geral de unicidade da solução, o que indica que o sistema pode ter mais de uma solução diferente. Geometricamente falando, mais de uma família de superfícies pode dar origem a uma mesma trajetória do sistema. O exemplo mais simples dessa possibilidade é o da partícula livre em três dimensões, cuja equação de HJ vem a ser

$$
\frac{\partial S}{\partial t}+\frac{1}{2 m}\left[\left(\frac{\partial S}{\partial x}\right)^{2}+\left(\frac{\partial S}{\partial y}\right)^{2}+\left(\frac{\partial S}{\partial x}\right)^{2}\right]=0 .
$$

Uma das soluções para a equação de HJ desse sistema pode ser encontrada por separação de variáveis, método que usaremos nos exemplos a seguir. Esta solução é dada pela função

$$
\begin{aligned}
& S(q, t)=-\frac{1}{2 m}\left(u_{x}^{2}+u_{y}^{2}+u_{z}^{2}\right) t \\
& +u_{x} x+u_{y} y+u_{z} z
\end{aligned}
$$

em que $u_{x}, u_{y}$ e $u_{z}$ são parâmetros. Esta função representa uma família de planos no espaço euclidiano.

Outra solução pode ser encontrada a partir da última das Eqs. (29), que pode ser resolvida por uma integração sobre $C$. Esta solução seria

$$
\begin{gathered}
S(q, t)=-\frac{m}{2 t}\left[\left(x-x_{0}\right)^{2}+\right. \\
\left.\left(y-y_{0}\right)^{2}+\left(z-z_{0}\right)^{2}\right],
\end{gathered}
$$

que representa uma família de esferas concêntricas, cuja origem é o ponto $\left(x_{0}, y_{0}, z_{0}\right)$.

Note que ambas as soluções dependem de três parâmetros arbitrários. Essa é uma característica das soluções, já que elas representam famílias de superfícies. No caso da partícula livre, cuja solução depende do tempo, uma determinada escolha de parâmetros implica na escolha de uma superfície inicial e a equação de HJ determina univocamente a evolução das superfícies. Uma solução completa da equação de HJ contém tantos parâmetros quantos forem os graus de liberdade do sistema.

Vamos supor que dispomos de uma solução particular da equação de HJ, ou seja, que depende de um número de parâmetros inferior ao número de graus de liberdade

$$
S=S\left(t, q^{1}, q^{2}, \ldots, q^{n}, u_{1}, u_{2}, \ldots, u_{m}\right),
$$

na qual $u_{l}$ são constantes, $\{l\}=\{1, \ldots, m\}$ e $m<n$. Então,

$$
\frac{d}{d t}\left(\frac{\partial S}{\partial u_{l}}\right)=\frac{\partial^{2} S}{\partial t \partial u_{l}}+\dot{q}^{i} \frac{\partial^{2} S}{\partial q^{i} \partial u_{l}} .
$$

A partir da Eq. (19),

$$
\frac{\partial^{2} S}{\partial t \partial u_{l}}=-\frac{\partial H}{\partial p_{i}} \frac{\partial^{2} S}{\partial q^{i} \partial u_{l}}=-\dot{q}^{i} \frac{\partial^{2} S}{\partial q^{i} \partial u_{l}},
$$

onde usamos a identidade da Eq. (22). Assim, da Eq. (36)

$$
\frac{d}{d t}\left(\frac{\partial S}{\partial u_{l}}\right)=0 \quad \longrightarrow \quad \frac{\partial S}{\partial u_{l}}=\beta^{l},
$$

onde $\beta^{l}$ são constantes. Assim, se dispusermos de uma solução parcial da equação de HJ dependente de $m<n$ constantes de integração, as derivadas de $S$ com relação a essas constantes serão constantes do movimento.

Considere agora a solução completa da equação de HJ

$$
S=S\left(t, q^{1}, q^{2}, \ldots, q^{n}, u_{1}, u_{2}, \ldots, u_{n}\right),
$$

em conjunto com $n$ constantes $\beta^{i}$. Devemos exigir também que a matriz cujos elementos são dados por

$$
\left(\frac{\partial^{2} S}{\partial q^{i} \partial u_{j}}\right)
$$

tenha determinante não nulo. Assim, as funções

$$
q^{i}=q^{i}\left(t, u_{1}, u_{2}, \ldots, u_{n}, \beta^{1}, \beta^{2}, \ldots, \beta^{n}\right),
$$

resultantes da equação

$$
\frac{\partial S}{\partial u_{i}}=\beta^{i}
$$

e as funções

$$
p_{i}=\frac{\partial S}{\partial q^{i}}=p_{i}\left(t, q^{1}, q^{2}, \ldots, q^{n}, u_{1}, u_{2}, \ldots, u_{n}\right),
$$

são soluções das equações canônicas (24). Este vem a ser o Teorema de Jacobi.

Vamos ver o que acontece às soluções da partícula livre. Para as ondas planas, a derivação da Eq. (32) com relação aos seus parâmetros, igualada a constantes, resulta na trajetória parametrizada

$$
\left\{\begin{array}{l}
x=\beta_{x}+\left(u_{x} / m\right) t, \\
y=\beta_{y}+\left(u_{y} / m\right) t, \\
z=\beta_{z}+\left(u_{z} / m\right) t .
\end{array}\right.
$$

As constantes $\beta$ são, neste caso, as coordenadas da posição inicial. Aplicando-se a Eq. (42), descobrimos que $p_{i}=u_{i}$, de modo que $u_{i} / m$ são as componentes da velocidade, que é constante ao longo da trajetória. 


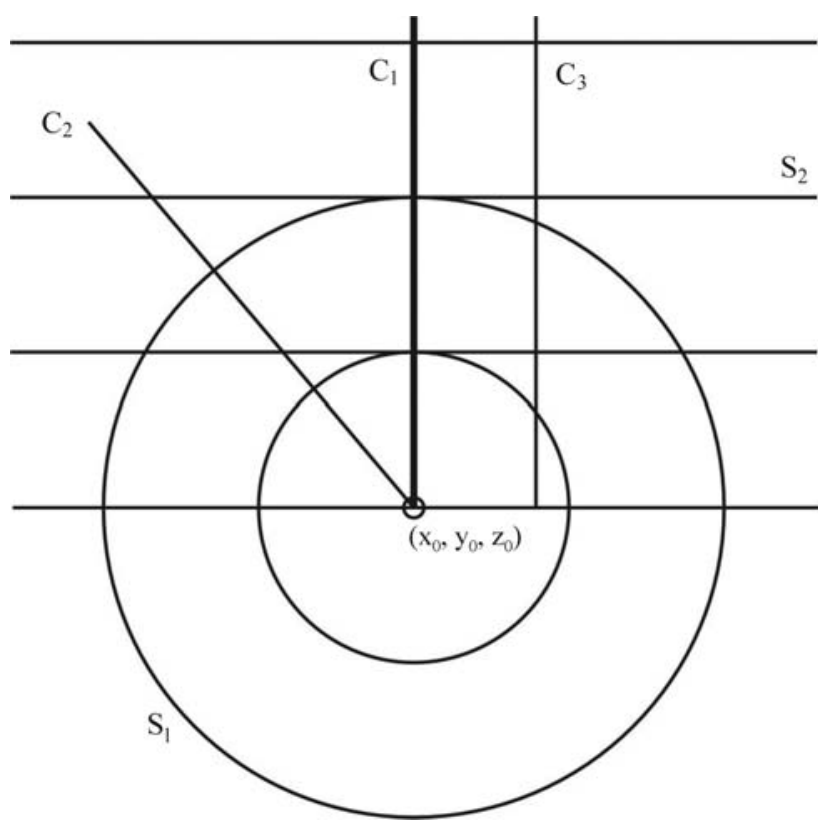

Figura 3 - Duas soluções da equação de HJ para a partícula livre. A solução $S_{1}$ representa uma família de esferas centradas no ponto $\left(x_{0}, y_{0}, z_{0}\right) . S_{2}$ é a solução de ondas planas. As trajetórias $C_{1}$ e $C_{2}$ são soluções encontradas pelo teorema de Jacobi para a família $S_{1}$, enquanto $C_{1}$ e $C_{3}$ são duas trajetórias que se referem à família $S_{2}$. Apenas $C_{1}$ é solução de ambas as famílias de superfícies.

A mesma análise pode ser feita com a solução que representa a família de ondas esféricas. As Eqs. (41) podem ser resolvidas para as coordenadas, de modo que

$$
\left\{\begin{array}{l}
x=x_{0}+\left(\beta_{x} / m\right) t \\
y=y_{0}+\left(\beta_{y} / m\right) t \\
z=z_{0}+\left(\beta_{z} / m\right) t
\end{array}\right.
$$

Os momentos são dados por $p_{i}=\beta_{i}$, como pode ser facilmente verificado pela derivação com relação às posições.

No entanto, as Eqs. (43) e (44) não representam a mesma família de curvas. Neste caso apenas uma trajetória é ortogonal às duas famílias de superfícies. No caso da primeira solução, a onda plana, as soluções da Eq. (43) representam um ensemble de partículas livres com velocidades distintas, mas de mesma direção. No segundo caso, a onda esférica, as soluções referem-se a um ensemble de partículas livres que partem de um mesmo ponto, $\left(x_{0}, y_{0}, z_{0}\right)$, mas que podem ter qualquer direção. Na Fig. 3 vemos que somente uma trajetória pode ser solução de ambas as famílias.

\section{Exemplos}

Vamos analisar dois exemplos de sistemas regulares para os quais é possível resolver diretamente a equação de HJ pelo método de separação de variáveis. Assim, vamos procurar por soluções na forma $S\left(t, q^{i}, u_{i}\right)$ resolvendo diretamente a equação diferencial parcial para esses problemas e assim seremos capazes de utilizar a solução para encontrar as equações de movimento. As equações características se reduzem às equações de Hamilton para esses sistemas, de modo que não há vantagem em utilizá-las no caso regular. A grande vantagem do método das características aparecerá para sistemas singulares.

\subsection{Oscilador harmônico unidimensional}

Vamos, neste exemplo, considerar o oscilador harmônico em uma dimensão. A grande maioria dos problemas envolvendo osciladores pode ser reduzida a resolver um único oscilador ou vários osciladores unidimensionais com freqüências angulares distintas. A Lagrangiana se escreve

$$
L=\frac{1}{2} m\left(\dot{x}^{2}-\omega^{2} x^{2}\right) .
$$

A matriz Hessiana deste sistema é regular. De fato, para este problema assim como para todo potencial independente das velocidades, a matriz é a mesma da partícula livre:

$$
\left(\frac{\partial^{2} L}{\partial \dot{x}^{2}}\right)_{1 \times 1}=m .
$$

Então, o sistema é regular. Isto significa que podemos escrever a velocidade em função do momento, $\dot{x}=p / m$, o que nos permite introduzir a Hamiltoniana

$$
H=\frac{1}{2 m} p^{2}+\frac{1}{2} m \omega^{2} x^{2}
$$

A Eq. (19) de HJ para essa Hamiltoniana é não homogênea:

$$
\frac{\partial S}{\partial t}+\frac{1}{2 m}\left(\frac{\partial S}{\partial x}\right)^{2}+\frac{1}{2} m \omega^{2} x^{2}=0
$$

Vamos separar esta equação, escrevendo a solução

$$
S=S_{0}(t)+W(x) .
$$

Substituindo a Eq. (48) na Eq. (47),

$$
\frac{d S_{0}}{d t}+\frac{1}{2 m}\left(\frac{d W}{d x}\right)^{2}+\frac{1}{2} m \omega^{2} x^{2}=0 .
$$

A Eq. (49) está completamente separada em suas variáveis. Neste caso, a expressão $d S_{0} / d t$ deve ser igual a alguma função independente de $x$. Por outro lado, a expressão

$$
\frac{1}{2 m}\left(\frac{d W}{d x}\right)^{2}+\frac{1}{2} m \omega^{2} x^{2}
$$

é igual a uma função independente de $t$. Dada a validade da Eq. (49), trata-se da mesma função, de modo que ambas as expressões não podem depender de $x$ ou t. São, portanto, constantes. Isso nos permite escrever duas equações

$$
\frac{d S_{0}}{d t}=-E
$$




$$
\frac{1}{2 m}\left(\frac{d W}{d x}\right)^{2}=E-\frac{1}{2} m \omega^{2} x^{2} .
$$

A constante $E$ será reconhecida como a energia total do sistema. A solução da Eq. (50), a menos de uma constante de integração que pode ser ignorada sem que se modifiquem as equações de movimento, é

$$
S_{0}(t)=-E t .
$$

Sistemas em que a Hamiltoniana não depende explicitamente do tempo têm a parte da solução que depende do tempo sempre na forma $S_{0}=-E t$. A Eq. (51) pode ser escrita em forma de quadratura

$$
W=\sqrt{2 m} \int\left(E-\frac{1}{2} m \omega^{2} x^{2}\right)^{\frac{1}{2}} d x .
$$

Para resolver a integral (53) vamos utilizar a seguinte substituição de variáveis

$$
a=\omega x \sqrt{\frac{m}{2 E}} .
$$

A Eq. (53) toma então a forma

$$
\begin{aligned}
W= & \frac{2 E}{\omega} \int\left(1-a^{2}\right)^{\frac{1}{2}} d a= \\
& \frac{2 E}{\omega}\left[\frac{a}{2}\left(1-a^{2}\right)^{1 / 2}+\frac{1}{2} \cos ^{-1} a\right] .
\end{aligned}
$$

A solução geral, em termos da variável $a$, vem a ser

$$
S=\frac{2 E}{\omega}\left[\frac{a}{2}\left(1-a^{2}\right)^{1 / 2}+\frac{1}{2} \cos ^{-1} a\right]-E t .
$$

Vamos procurar pelo momento

$$
p=\frac{\partial S}{\partial x}=\frac{\partial S}{\partial a} \frac{d a}{d x} .
$$

Note que

$$
\frac{\partial S}{\partial a}=\frac{2 E}{\omega} \frac{\partial}{\partial a} \int\left(1-a^{2}\right)^{\frac{1}{2}} d a=\frac{2 E}{\omega}\left(1-a^{2}\right)^{\frac{1}{2}} .
$$

Dessa forma, usando as Eqs. (54) e (57),

$$
p=\sqrt{2 m}\left(E-\frac{1}{2} m \omega^{2} x^{2}\right)^{1 / 2} .
$$

Essa equação nos faz reconhecer $E$ como a energia. Agora, a derivada com relação a $E$

$$
\begin{aligned}
& \beta=\frac{\partial S}{\partial E}=\left.\frac{\partial S}{\partial E}\right|_{a=c t e}+\left.\frac{d a}{d E} \frac{\partial S}{\partial a}\right|_{E=c t e}= \\
& -t+\frac{2}{\omega} \int\left(1-a^{2}\right)^{\frac{1}{2}} d a-\frac{a}{\omega}\left(1-a^{2}\right)^{1 / 2}= \\
& -t+\frac{2}{\omega}\left[\frac{a}{2}\left(1-a^{2}\right)^{1 / 2}+\frac{1}{2} \cos ^{-1} a\right] \\
& -\frac{a}{\omega}\left(1-a^{2}\right)^{1 / 2}=-t+\frac{1}{\omega} \cos ^{-1} a .
\end{aligned}
$$

Desta equação temos

$$
x=\frac{1}{\omega} \sqrt{\frac{2 E}{m}} \cos \omega(t+\beta),
$$

ou, em forma mais familiar,

$$
x=A \cos \omega\left(t-t_{0}\right)
$$

$\operatorname{com} A=(1 / \omega) \sqrt{2 E / m}$ e $t_{0}=-\beta$. A Eq. (62) vem a ser a conhecida solução do oscilador harmônico unidimensional.

\subsection{Partícula carregada em campo magnético}

Neste segundo exemplo, vamos considerar uma partícula de carga $e$ que se move no plano $(x, y)$, submetida a um campo magnético constante na direção $z$. A função Lagrangiana de uma partícula em um campo eletromagnético é dada por

$$
L(x, y, \dot{x}, \dot{y})=\frac{1}{2} m\left(\dot{x}^{2}+\dot{y}^{2}\right)-e \psi+e \mathbf{v} \cdot \mathbf{A},
$$

em que $\psi=\psi(x, t)$ é o potencial escalar e os vetores $\mathbf{v}$ e $\mathbf{A}(x, t)$ são, respectivamente, a velocidade da partícula e o potencial vetor eletromagnético. A tem, no geral, componentes nas direções $x$ e $y$, mas uma escolha apropriada de gauge nos permite escrever este vetor como tendo apenas uma componente na direção y (gauge de Landau). Assim, podemos escrever $A_{y}=x B$. O potencial escalar é nulo neste exemplo. Ficaremos, então, com a Lagrangiana

$$
L(x, y, \dot{x}, \dot{y})=\frac{1}{2} m\left(\dot{x}^{2}+\dot{y}^{2}\right)+e B x \dot{y} .
$$

Os momentos podem ser calculados e são ambos inversíveis

$$
p_{x} \equiv \frac{\partial L}{\partial \dot{x}}=m \dot{x} \quad \longrightarrow \quad \dot{x}=\frac{p_{x}}{m},
$$

$p_{y} \equiv \frac{\partial L}{\partial \dot{y}}=m \dot{y}+e B x \quad \longrightarrow \quad m \dot{y}=p_{y}-e B x$.

Para construir a Hamiltoniana, usaremos a Eq. (18)

$$
H\left(x, y, p_{x}, p_{y}\right)=\frac{1}{2 m}\left[\left(p_{x}\right)^{2}+\left(p_{y}-e B x\right)^{2}\right] .
$$

Temos a EDP

$$
2 m \frac{\partial S}{\partial t}+\left(\frac{\partial S}{\partial x}\right)^{2}+\left(\frac{\partial S}{\partial y}-e B x\right)^{2}=0
$$

Neste problema usaremos a seguinte separação de variáveis

$$
S(t, x, y)=S_{0}(t)+W_{x}(x)+W_{y}(y) .
$$


A primeira solução vem a ser a já conhecida $S_{0}=-E t$, com a qual podemos reescrever a equação como

$$
\left(\frac{d W_{x}}{d x}\right)^{2}+\left(\frac{d W_{y}}{d y}-e B x\right)^{2}=2 m E .
$$

Esta equação ainda não está numa forma separável. Contudo, podemos isolar as partes dependentes de $x$ e $y$ da seguinte forma

$$
\frac{d W_{y}}{d y}=e B x+\sqrt{2 m E-\left(\frac{d W_{x}}{d x}\right)^{2}} .
$$

A Eq. (67) está completamente separada. Portanto, cada lado da equação deve ser igual a uma constante. Temos, então, duas equações

$$
\begin{gathered}
\frac{d W_{y}}{d y}=\alpha, \\
\frac{d W_{x}}{d x}=\sqrt{2 m E-(\alpha-e B x)^{2}} .
\end{gathered}
$$

Para $W_{y}$ já podemos escrever

$$
W_{y}(y)=\alpha y .
$$

Para $W_{x}$ a solução é a quadratura

$$
W_{x}(x)=\int \sqrt{2 m E-(\alpha-e B x)^{2}} d x .
$$

Não precisamos resolver a quadratura para aplicar o teorema de Jacobi, pois precisamos das derivadas das soluções, e não da solução em si. Como solução completa escrevemos

$$
\begin{gathered}
S(t, x, y)=-E t+\alpha y+ \\
\int \sqrt{2 m E-(\alpha-e B x)^{2}} d x .
\end{gathered}
$$

Temos

$$
\begin{array}{r}
p_{x}=\frac{\partial S}{\partial x}=\sqrt{2 m E-(\alpha-e B x)^{2}} \mathrm{e} \\
p_{y}=\frac{\partial S}{\partial y}=\alpha .
\end{array}
$$

Esses são os momentos nas direções $x$ e $y$. O momento em $y$ é a constante de separação $\alpha$ mas o momento $p_{x}$ depende de $x$. Vamos procurar pelas equações de movimento. Existem duas constantes, $E$ e $\alpha$. A derivada com relação a $E$ é

$$
\frac{\partial S}{\partial E}=-t+m \int \frac{d x}{\sqrt{2 m E-(\alpha-e B x)^{2}}}=\beta_{1} .
$$

A integral pode ser efetuada por uma substituição trigonométrica. A solução é

$$
\begin{gathered}
\int \frac{d x}{\sqrt{2 m E-(\alpha-e B x)^{2}}}= \\
-\frac{1}{e B} \sin ^{-1}\left(\frac{\alpha-e B x}{\sqrt{2 m E}}\right) .
\end{gathered}
$$

Temos, portanto, a primeira equação

$$
t+\beta_{1}=-\frac{m}{e B} \sin ^{-1}\left(\frac{\alpha-e B x}{\sqrt{2 m E}}\right),
$$

que pode ser resolvida para $x$ :

$$
x(t)=\frac{\alpha}{e B}-\frac{\sqrt{2 m E}}{e B} \sin \left[-\frac{e B}{m}\left(t+\beta_{1}\right)\right] .
$$

Essa equação de movimento toma uma forma mais familiar

$$
x(t)=x_{0}+r \sin \left[\omega_{0}\left(t-t_{0}\right)\right],
$$

$\operatorname{com} x_{0}=\alpha / e B, r=\sqrt{2 m E} / e B, \omega=e B / m$ e $t_{0}=-\beta_{1}$. Essa equação dá a dinâmica do sistema na direção $x$, que vem a ser um movimento oscilatório com centro em $x_{0}$, amplitude $r$ e freqüência angular $\omega_{0}$. Falta ainda a equação para a direção $y$, que deve vir da derivada de $S$ com relação a $\alpha$

$$
\frac{\partial S}{\partial \alpha}=y-\int \frac{(\alpha-e B x) d x}{\sqrt{2 m E-(\alpha-e B x)^{2}}}=\beta_{2} .
$$

Essa integral tem solução

$$
\begin{aligned}
& \int \frac{(\alpha-e B x) d x}{\sqrt{2 m E-(\alpha-e B x)^{2}}}= \\
& \frac{1}{e B} \sqrt{2 m E-(\alpha-e B x)^{2}},
\end{aligned}
$$

de modo que temos a equação

$$
y-\beta_{2}=\frac{1}{e B} \sqrt{2 m E-(\alpha-e B x)^{2}} .
$$

Esta equação não é dinâmica, mas com alguma manipulação podemos colocá-la na forma

$$
\left(y-\beta_{2}\right)^{2}+\left(x-\frac{\alpha}{e B}\right)^{2}=\frac{2 m E}{e^{2} B^{2}},
$$

ou

$$
\left(y-y_{0}\right)^{2}+\left(x-x_{0}\right)^{2}=r^{2},
$$

$\operatorname{com} y_{0}=\beta_{2}$. Essa equação dá a trajetória no espaço de configuração, que é um círculo de raio $r$ com centro no ponto $\left(x_{0}, y_{0}\right)$. De fato, essa trajetória é uma família de circunferências de raios distintos, já que o raio depende arbitrariamente da energia do sistema. As Eqs. (78) e (83) são suficientes para determinar a dinâmica do sistema ${ }^{8}$.

${ }^{8}$ Para determinar a equação temporal para y basta substituir a Eq. (78) na Eq. (83) e resolver para y. O resultado também será um movimento oscilatório com freqüência angular $\omega_{0}$. 
O fato de o momento na direção y ser uma constante pode gerar confusão quando analisamos as soluções, pois $y$ não varia linearmente com o tempo, mas de forma oscilatória. Contudo, esse momento não pode ser confundido com a componente vetorial $m \dot{y}$. O momento dado pelas Eqs. (73) depende da escolha de gauge, sendo este dado por $\mathbf{p}=m \dot{\mathbf{x}}-e \mathbf{A}$. No gauge escolhido neste exemplo, o gauge de Landau, o momento $p_{x}$ de fato coincide com a componente $m \dot{x}$, mas isso não ocorre com $p_{y}$. Este é um exemplo no qual a trajetória dinâmica dada pela solução (83) não é ortogonal à família de superfícies definida por $S$. Apenas a direção do momento conjugado, dado por $\partial L / \partial \dot{q}$, é ortogonal.

Nossa escolha de gauge foi feita para facilitar a separação da equação de HJ. No entanto, a escolha de gauge não interfere na dinâmica do sistema. A escolha poderia ter sido outra, o que resultaria em outra equação diferencial para $S$, talvez mais difícil de separar, ou mesmo impossível. Assim, quando o sistema possui uma simetria de gauge, existem infinitas equações de HJ que podem ser resolvidas, mas todas devem resultar na mesma trajetória dinâmica no fim do processo. A separabilidade do sistema depende, portanto, da escolha de gauge, bem como da escolha de um sistema de coordenadas apropriado.

\section{Considerações finais}

Nesse trabalho apresentamos a versão de Carathéodory do formalismo de Hamilton-Jacobi e vimos como estão relacionados o cálculo variacional, a teoria das equações diferenciais parciais de primeira ordem e a teoria das equações diferenciais ordinárias, no quadro completo de Carathéodory. Obviamente exploramos apenas de forma introdutória toda a riqueza que há por trás desse ponto de vista. Muitos aspectos, que representam partes fundamentais do formalismo de HJ, não foram tratados aqui. Vamos tecer alguns comentários sobre esses aspectos.

O parênteses de Poisson de dois observáveis é uma função escalar do espaço de fase, o que significa que ele é invariante a uma certa classe de transformação de coordenadas. Essa classe de transformações são as transformações canônicas (TC), que têm a propriedade de deixar covariantes as equações de movimento.

No programa usual da mecânica clássica, ${ }^{9}$ o formalismo Hamiltoniano emerge do formalismo Lagrangiano a partir de uma transformação de Legrendre. O formalismo de Hamilton-Jacobi, por outro lado, é derivado do formalismo Hamiltoniano quando procuramos por uma transformação canônica que anule a função de Hamilton. Quando uma transformação canônica é feita sobre o espaço de fase, existe uma função, chamada de geratriz, que contém toda informação sobre a TC, no sentido de que ela recupera as equações de movimento nas antigas e nas novas variáveis. A transformação gera uma nova Hamiltoniana $K$, que se relaciona com $H$ a partir da equação $K=H+\partial S / \partial t$, onde $S$ é a função geratriz da TC. Quando exigimos que $K=0$, recuperamos a equação de HJ.

As soluções da equação de HJ são as geratrizes dessa transformação canônica e, como toda geratriz, essas soluções devem depender das antigas e das novas variáveis do espaço de fase. Podemos observar, por exemplo, a solução de ondas planas da partícula livre (Eq. (34)). Ela depende das três componentes da posição da partícula e, também, de três parâmetros arbitrários. Essa função é geratriz de uma transformação canônica que transforma os momentos em constantes. Já a função de ondas esféricas da Eq. (35) é geratriz de uma transformação que leva as posições a constantes. São tipos diferentes de transformações que resultam na mesma equação de HJ. Jacobi foi o autor da teoria das transformações canônicas e, sob esta ótica, desenvolveu também o formalismo de Hamilton-Jacobi, mas não sozinho, pois Hamilton já havia dado os primeiros passos nessa direção, baseado na analogia óptico-mecânica.

Outro aspecto muito importante é o fato de que a ação $A$, calculada como a integral indefinida $A\left(q, q_{0}, t, t_{0}\right)=\int L d t$, é uma solução da equação de HJ. De fato, a trajetória dinâmica representada pela curva $C$ no espaço de fase pode ser vista como uma sucessão de transformações canônicas infinitesimais a partir de um estado inicial $\left(q_{0}, p_{0}\right)$. A ação é uma função geratriz dessa transformação e ela obedece à equação de HJ. A última das equações características (29), quando resolvida para $S$, é exatamente a integral da ação em sua forma canônica $S=\int\left(p_{i} \dot{q}^{i}-H\right) d t$.

Por fim, devemos ter em mente que uma solução completa da equação de HJ deve ser encontrada para que se possa, seja pelo método das características ou pela resolução em si da equação, encontrar as curvas características do sistema. Não há, a princípio, nenhuma garantia de que exista uma solução completa de um dado sistema de equações diferenciais parciais, de modo que condições de integrabilidade deveriam ser impostas à equação de HJ. Se escrevermos $\Phi_{0} \equiv \partial S / \partial t+H$, a condição para que exista uma solução completa da equação $\Phi_{0}=0$ é dada por $\dot{\Phi}_{0}=0$. Essa condição, contudo, é identicamente satisfeita para o caso regular, como pode ser facilmente verificado com o uso dos parênteses de Poisson. Assim, todo sistema cuja matriz Hessiana tem determinante não nulo possui uma solução completa da equação de HJ.

Há, todavia, inúmeros casos em que a o determinante da matriz Hessiana é nulo, dentre os quais se encontram inúmeros problemas tratados em Teoria de Campos. São os chamados sistemas singulares. Estes casos não podem ser estudados nesta formulação à la Carathéodory, conforme desenvolvida aqui. Durante muitos anos o tratamento de sistemas singulares

\footnotetext{
${ }^{9}$ Por exemplo, Ref. [4].
} 
no contexto de HJ ficou como um problema em aberto, e estes eram estudados apenas no formalismo hamiltoniano, conforme procedimento estabelecido por Dirac nos anos 1950 [12-14]. Os primeiros trabalhos estendendo a formulação desenvolvida por Carathéodory para o tratamento de sistemas singulares foram feitos por Güler em 1992 [15, 16]. Outras extensões foram realizadas por outros autores e, hoje, é bem estabelecido como são descritos em HJ os sistemas singulares com derivadas de ordem superior a um [24, 25], sistemas singulares descritos por variáveis de Berezin [26], sistemas descritos por ações de primeira ordem [27], entre outros. Desde o desenvolvimento inicial feito por Güler, diversas aplicações a sistemas singulares têm sido realizadas [17-23], provendo um novo olhar no estudo de sistemas singulares.

\section{Agradecimentos}

M.C. Bertin agradece à CAPES pelo suporte integral. B.M. Pimentel agradece ao $\mathrm{CNPq}$ e à FAPESP pelo suporte parcial. P.J. Pompeia agradece ao CTA pelo incentivo e suporte.

\section{Referências}

[1] E. Schrödinger, Collected Papers on Wave Mechanics (Chelsea Pub. Co., Nova Iorque, 1982).

[2] M. Kline, Mathematical Thought From Ancient to Modern Times (Oxford University Press, New York, 1990), v. 2.

[3] C. Carathéodory, Calculus of Variations and Partial Differential Equations of the First Order - Part I, II (Holden Day, Inc, San Francisco, 1967).

[4] H. Goldstein, Classical Mechanics (Addison-Wesley, Reading, 1950).

[5] E. Whittaker, A Treatise on the Analytical Dynamics of Particles and Rigid Bodies (Cambridge University Press, Cambrige, 1959).

[6] L. Landau and E. Lifchitz, Mecânica - (Hemus, São Paulo, 2004).
[7] W. Wreszinski, Mecânica Clássica Moderna (Edusp, São Paulo, 1997).

[8] C. Lanczos, The Variational Principles of Mechanics (Dover Pub. INC., Nova Iorque, 1986), 4th ed, cap. VIII.

[9] J.V. José e E.J. Saletan, Classical Dynamics, A Contemporary Approach (Cambridge University Press, Cambrige, 1998), cap. 6.

[10] I.M. Gelfand e S.V. Fomin, Calculus of Variations (Dover Pub. INC., Nova Iorque, 2000).

[11] R. Courant e D. Hilbert, Methods of Mathematical Physics (John Wiley and Sons, Nova Iorque, 1962), v. 2.

[12] P.A.M. Dirac, Can. J. Math. 2, 129 (1950).

[13] P.A.M. Dirac, Can. J. Math. 3, 129 (1951).

[14] P.A.M. Dirac, Lectures on Quantum Mechanics (Yeshiva University, New York, 1964).

[15] Y. Güler, Il Nuovo Cimento B 107, 1398 (1992).

[16] Y. Güler, Il Nuovo Cimento B 107, 1143 (1992).

[17] Y. Güler, Il Nuovo Cimento B 109, 341 (1994).

[18] Y. Güler, Il Nuovo Cimento B 111, 513 (1996).

[19] Y. Güler and D. Baleanu, Il Nuovo Cimento B 114, 1023 (1999).

[20] Y. Güler and D. Baleanu, Il Nuovo Cimento B 115, 319 (2000).

[21] Soon-Tae Hong, Yong-Wan Kim, Young-Jai Park and K.D. Rothe, Mod. Phys. Lett. A 17, 435 (2002).

[22] B.M. Pimentel, P.J. Pompeia, J.F. da Rocha-Neto and R.G. Teixeira, Gen. Rel. Grav. 35, 877 (2003).

[23] B.M. Pimentel, P.J. Pompeia e J.F. da Rocha-Neto, Il Nuovo Cimento B 120, 981 (2005).

[24] B.M. Pimentel and R.G. Teixeira, Il Nuovo Cimento B 111, 841 (1996).

[25] B.M. Pimentel and R.G. Teixeira, Il Nuovo Cimento B 113, 805 (1998).

[26] B.M. Pimentel, R.G. Teixeira and J.L. Tomazelli, Ann. Phys. 267, 75 (1998).

[27] M.C. Bertin, B M. Pimentel and P.J. Pompeia, Mod. Phys. Lett. A 20, 2873 (2005). 